\title{
Reply to "Discussion to 'Boussinesq modeling of longshore currents in the Sandy Duck experiment under directional random wave conditions' by J. Choi, J. T. Kirby and S.B. Yoon"
}

\author{
Junwoo Choi $^{1}$, James T. Kirby ${ }^{2 *}$ and Sung Bum Yoon ${ }^{3}$ \\ ${ }^{1}$ River and Coastal Research Division, Korea Institute of Civil Engineering $\mathcal{E} 3$ Building \\ Technology, Goyang 411-712, South Korea \\ ${ }^{2}$ Center for Applied Coastal Research, Department of Civil and Environmental \\ Engineering, University of Delaware, Newark, DE 19716 USA \\ ${ }^{3}$ Department of Civil and Environmental Engineering, Hanyang University, Ansan, \\ 426-791, South Korea \\ ${ }^{*}$ Corresponding author: Tel: 1-302-831-2438; Email address: kirby@udel.edu
}

$1 \quad$ Simaro makes the interesting (and previously known) point that the 2 choice of $z_{\alpha} / h=-0.53$ proposed by Nwogu does not provide an across3 the-board optimization of all linear properties of the Boussinesq equations. 4 In particular, Madsen and Sorensen (1992) have shown that this choice can 5 lead to apparent errors in linear shoaling properties over the range of frequen6 cies for which the phase speed optimization is computed. Simarro (2013) has 7 further investigated these effects and has developed a more compact means 8 for estimating a value for $z_{\alpha} / h$ that minimizes a global error estimate based 9 on both phase speed and shoaling properties.

10 While we do not contest that the choice of $z_{\alpha} / h$ proposed by Simarro 11 represents a potentially more optimal linear model than the across-the-board 12 use of Nwogu's original value, it is not clear at the outset how the choice of 
${ }_{13} z_{\alpha} / h$ plays out in the computation of a realistic random wave train. When

considering waves approaching breaking, under- or overshoaling errors would presumably lead to a displacement of wave breaking slightly on or offshore of the correct location. Whether this effect is detectable in a sequence of random waves, and whether it has consequences for the details of surf zone circulation, is unknown.

In order to explore the consequences of the choice of $z_{\alpha} / h$, we carry out additional computations here on the simpler case of shoaling and breaking of a normally incident wave train in 1-D. This choice eliminates the complicating effect of breaking wave short-crestedness, which we would a priori expect to be the dominant factor in the 2-D case. (see the paper under discussion as well as Feddersen et al., 2014). The data set used is due to Mase and Kirby (1992), who considered the shoaling and evolution of two TMA-type spectra over a smooth, planar beach at laboratory scale. This data set is provided as part of the set of installation tests for Funwave (available at http://www.udel.edu/kirby/programs/funwave/funwave.html), and has been used extensively to test a range of models including other Boussinesq codes as well as nonhydrostatic codes (Ma et al., 2012). The Funwave code used here is Version 2.0 of Funwave-TVD, described by Shi et al. (2012). (Choi et al. (2015) used the older finite-difference code described by Kennedy et al. (2000) and Chen et al. (2000), but this difference is not expected to be significant.) The program was compiled as a serial code using gfortran, and run on a Mac Pro. The code as written allows the user to select the 
value of $z_{\alpha} / h$ in the input data. Here, we use values of $z_{\alpha} / h$ corresponding to optimization for phase speed (following Nwogu, 1993) and linear shoaling coefficient (following Simarro, 2013). The data set used for comparison and for model boundary data is Run 2 of Mase and Kirby (1992). The data set as well as all needed pre- and postprocessing software is provided as part of the Funwave installation; additional Matlab code used to compare both model results to data may be obtained on request from the second author. The model is set up with the wavemaker at the location of the wave gauge in 47 $\mathrm{cm}$ water depth and the model is driven by measured data at that location. Consequently, computations are not reported at this depth since the gauge lies inside the wave generation region in the model.

Two model runs are considered below. The first uses the standard choice of $z_{\alpha} / h=-0.53$ as specified in Nwogu (1993). The second uses a value of $z_{\alpha} / h=-0.55$, which is chosen corresponding to the large range of $k h$ values represented in the incident wave train, following the guidance in Table 1 of Simarro (2015). (We did not explore the use of larger values of $z_{\alpha} / h$, which could potentially better optimize the problem for frequencies near the wave train's peak frequency). Results for water surface displacements $\eta(t)$, significant wave height $H_{s}=4.002 m_{0}^{1 / 2}$, normalized skewness and asymmetry, and power spectral densities $S_{\eta \eta}(f)$ are shown in Figures (1) - (4), respectively. 56 Normalized skewness $\mathcal{S}$ and asymmetry $\mathcal{A}$, which quantify the importance of 
nonlinearity in adjusting wave shape, are defined by

$$
\mathcal{S}=\left\langle(\eta-\langle\eta\rangle)^{3}\right\rangle /\left\langle(\eta-\langle\eta\rangle)^{2}\right\rangle^{3 / 2} ; \quad \mathcal{A}=\left\langle\mathcal{H}(\eta-\langle\eta\rangle)^{3}\right\rangle /\left\langle(\eta-\langle\eta\rangle)^{2}\right\rangle^{3 / 2}
$$

where $\mathcal{H}()$ denotes the Hilbert transform of the argument and \langle\rangle denotes an average. Power spectral densities are computed using Welch's method with a window size of 512 points, using a cosine taper with no overlap. Data processing procedures for measured and model results are identical.

The results show that computations based on the two optimization choices are generally in better agreement with each other than either is with the data. Overall, results are comparable to previous computations for this case appearing in the literature. Deviations between model results and data can be traced to factors that are not accounted for in the model setup, which was done quickly and without great attention to detail. In particular, the presence of too much energy in low frequencies in the model results (Figure 4) is largely due to the fact that the measured data at $h=47 \mathrm{~cm}$ is interpreted entirely as waves which are progressive towards shore, whereas, in reality, there is a strong reflected component of energy at low frequencies leading to standing wave behavior. Errors in modeled wave heights, with model results uniformly underpredicted during shoaling transformation, are due partly to the use of a linearized wavemaker condition in relatively shallow water, with no attempts being made to adjust model input in order to rescale wave height at the first measuring location. No attempt was made to adjust wavemaker 
source width or default sponge layer dimensions during the simulations.

78

Although we agree that the careful optimization of model properties, as suggested by Simarro, can be a valuable exercise towards understanding the characteristics of model equations, the results here show that the changes in model computations resulting from such an optimization can be masked in results for spectral sea states to a degree that potentially eliminates the benefit of the optimization. We thus do not believe that failing to carry out such an effort is likely to cause any consistent discrepancies that would be detectable in results for the cases in the original paper. Any such changes are also more than likely to further be masked by factors such as uncertainty in bathymetric data, incident wave conditions and other local and remote forcing mechanisms such as tide and wind, that would be ubiquitous under field conditions.

\section{Acknowledgements}

In addition to support acknowledged in the original paper, Kirby would like to acknowledge present support from the National Science Foundation, Physical Oceanography Program (OCE-1435147)

\section{References}

Chen, Q., Kirby, J.T., Dalrymple, R.A., Kennedy, A.B., Chawla, A., 2000, "Boussinesq modeling of wave transformation, breaking and runup II: two horizontal dimensions", J. Waterway Port Coast. Ocean Eng., 126 (1), $48-56$. 
Choi, J., Kirby, J. T. and Yoon, S. B., 2015, "Boussinesq modeling of longshore currents in the SandyDuck experiment under directional random wave conditions", Coastal Engineering, 101, 17-34, doi:10.1016/ j.coastaleng.2015.04.005

Feddersen, F., 2014, "The generation of surfzone eddies in a strong alongshore current", J. Phys. Oceanogr. 44, 600-617.

Kennedy, A.B., Chen, Q., Kirby, J.T., Dalrymple, R.A., 2000, "Boussinesq modeling of wave transformation, breaking, and runup. I: 1D", J. Waterway Port Coast. Ocean Eng, 126, 39-47.

Ma, G., Shi, F. and Kirby, J. T., 2012, "Shock-capturing non-hydrostatic model for fully dispersive surface wave processes", Ocean Modelling, 43-44, 22-35.

Madsen, P. A. and Sorensen, O. R., 1992, "A new form of the Boussinesq equations with improved linear dispersion characteristics. Part 2. A slowly-varying bathymetry", Coastal Engineering, 18, 183-204.

Mase, H. and Kirby, J. T., 1992, "Modified frequency domain KdV equation for random wave shoaling", Proc. 23d Intl. Conf. Coastal Engrng., Venice, October, 474-487.

Nwogu, O., 1993, "Alternative form of Boussinesq equatios for nearshore wave propagation", Journal of Waterway, Port, Coastal and Ocean Engineering, 119, 618-638. 
120

121

122

123

124

125

126

127

128

129

130

Shi, F., Kirby, J. T., Harris, J. C., Geiman, J. D. and Grilli, S. T., 2012, "A high-order adaptive time-stepping TVD solver for Boussinesq modeling of breaking waves and coastal inundation", Ocean Modelling, 43-44, $36-51$.

Simarro, G., 2013, "Energy balance, wave shoaling and group celerity in Boussinesq-type wave propagation models", Ocean Modelling, 72, 7479, doi:10.1016/j.ocemod.2013.08.004

Simarro, G., 2015, "Discussion of 'Boussinesq modeling of longshore currents in the Sandy Duck experiment under directional random waves' by J. Choi, J. T. Kirby and S. B. Yoon", submitted to Coastal Engineering 


\section{List of Figures}

1 Sample water surface displacements at wave gauge locations. Mase and Kirby (1992), Run 2. Measured (solid), Nwogu optimization (dashed), Simarro optimization (dotted). . . . . 9

2 Significant wave height at wave gauge locations. Mase and Kirby (1992), Run 2. Measured (solid), Nwogu optimization (dashed), Simarro optimization (dotted). . . . . . . . 10

3 Normalized skewness (o) and - asymmetry (x) at wave gauge locations. Mase and Kirby (1992), Run 2. Measured (solid), Nwogu optimization (dashed), Simarro optimization (dotted). 11

4 Power spectra of surface displacements at wave gauge locations. Mase and Kirby (1992), Run 2. Shaded gray area shows $\pm 95 \%$ confidence limits for measured data. Nwogu optimization (dashed), Simarro optimization (dotted). . . . . . . . 12 
Figure 1: Sample water surface displacements at wave gauge locations. Mase and Kirby (1992), Run 2. Measured (solid), Nwogu optimization (dashed), Simarro optimization (dotted).

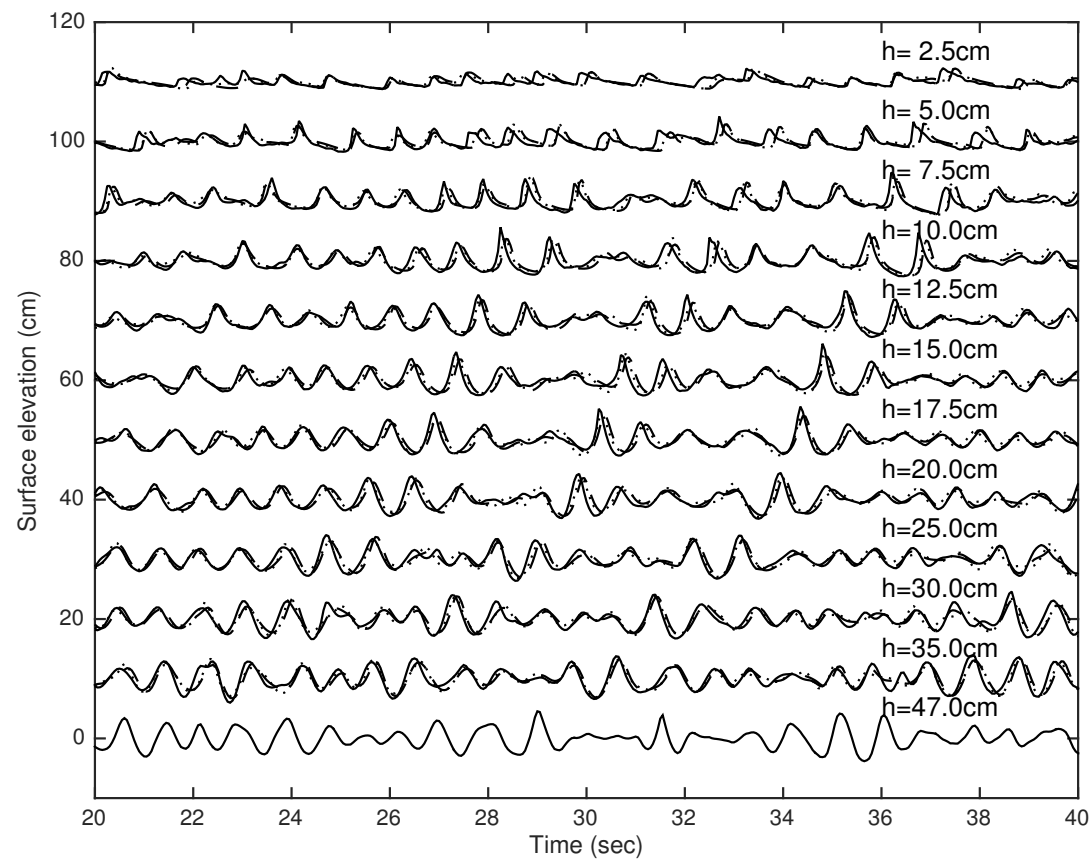


Figure 2: Significant wave height at wave gauge locations. Mase and Kirby (1992), Run 2. Measured (solid), Nwogu optimization (dashed), Simarro optimization (dotted). 
Figure 3: Normalized skewness (o) and - asymmetry (x) at wave gauge locations. Mase and Kirby (1992), Run 2. Measured (solid), Nwogu optimization (dashed), Simarro optimization (dotted). 
Figure 4: Power spectra of surface displacements at wave gauge locations. Mase and Kirby (1992), Run 2. Shaded gray area shows $\pm 95 \%$ confidence limits for measured data. Nwogu optimization (dashed), Simarro optimization (dotted). 